\title{
Explicit symplectic integrator for particle tracking in $s$-dependent static electric and magnetic fields with curved reference trajectory
}

\author{
A. Wolski ${ }^{*}$ and A. T. Herrod \\ University of Liverpool, Liverpool, United Kingdom and Cockcroft Institute, Warrington, United Kingdom
}

(Received 18 April 2018; published 13 August 2018)

\begin{abstract}
We describe a method for symplectic tracking of charged particles through static electric and magnetic fields. The method can be applied to cases where the fields have a dependence on the longitudinal as well as transverse position and where the reference trajectory may have nonzero curvature. Application of the method requires analytical expressions for the scalar and vector potentials: We show how suitable expressions, in the form of series analogous to multipole expansions, can be constructed from numerical field data, allowing the method to be used in cases where only numerical field data are available.
\end{abstract}

DOI: 10.1103/PhysRevAccelBeams.21.084001

\section{INTRODUCTION}

The magnetic and (in some cases) electric fields used to guide particles in an accelerator are often arranged so that particles ideally follow a curved trajectory. In simple cases, for example, a magnetic dipole field, standard expressions can be used to calculate the path of a particle through both the main field and the fringe-field regions of the relevant element. However, in more complex cases, calculating particle trajectories can be challenging: Such cases include, for example, situations where quadrupole or higher-order multipole fields are included by design within a dipole field or where account needs to be taken of multipole components occurring from systematic or random errors within the element. In general, the problem of particle tracking can be broken down into two parts. First, an accurate description of the field is needed; second, the equations of motion through the field must be integrated to find the path followed by a given particle. It is often possible to use a numerical field map to describe the field; then, standard integration algorithms (for example, Runge-Kutta algorithms) can be used to integrate the equations of motion. However, an approach such as this can be computationally expensive, both in terms of the memory needed to store the field data and in terms of the processing involved in integrating the equations of motion. Furthermore, if there are specific constraints or requirements for the trajectories, then additional challenges can occur. For example, if the tracking must obey the symplectic condition, then an

\footnotetext{
a.wolski@liverpool.ac.uk
}

Published by the American Physical Society under the terms of the Creative Commons Attribution 4.0 International license. Further distribution of this work must maintain attribution to the author(s) and the published article's title, journal citation, and DOI. explicit Runge-Kutta integration algorithm cannot be used. Symplectic Runge-Kutta algorithms do exist but are implicit in the sense that each step requires the solution of a set of algebraic equations that can add significantly to the computation time.

Regarding the description of the field, an alternative approach to a numerical field map is to represent the field as a superposition of a number of "modes." Given a set of coefficients, the field can be calculated at any position by summing the functions describing the different modes. This is the approach generally taken for multipole fields, for example, where the horizontal and vertical magnetic field components $B_{x}$ and $B_{y}$ (respectively) are given by

$$
B_{y}+i B_{x}=\sum_{m=0}^{m_{\max }} C_{m}(x+i y)^{m} .
$$

The upper limit of the sum, $m_{\max }$, is chosen to provide the accuracy required for the field. The advantages of this approach over a numerical field map are first that the data describing the field are contained in a relatively small set of coefficients and second that the calculation of the field at an arbitrary point does not need interpolation between grid points, which can be an issue in some circumstances for a numerical field map. The field represented by the multipole expansion (1) is independent of the distance along the reference trajectory and so is appropriate for the main field region within an accelerator element. Depending on the situation being considered, fringe fields may be neglected altogether (as in the "hard edge" approximation) or may be represented using appropriate expressions based, for example, on generalized gradients [1] or formulas representing solutions to Maxwell's equations with appropriate limiting behavior [2].

A semianalytical field description such as (1) has a further advantage over a purely numerical description in the 
context of particle tracking. In some cases, it is possible to construct explicit transfer maps parameterized, for example, in terms of the mode coefficients and element length: The transfer maps then offer the possibility of greater computational efficiency over numerical integration techniques, such as Runge-Kutta algorithms. Furthermore, if the transfer maps are constructed in an appropriate way, then the tracking can satisfy requirements such as symplecticity. An explicit symplectic integrator for general $s$ dependent static magnetic fields, in systems with a straight reference trajectory, has been presented by Wu, Forest, and Robin [3]. The application of the integrator requires the derivatives of the vector potential; it is therefore convenient to have a semianalytical field description, which allows the derivatives to be expressed in terms of appropriate modes in the same way as the potential itself, thus avoiding the need for taking derivatives numerically.

In elements designed to bend the beam trajectory, it is usually convenient to use a reference trajectory that follows the intended curvature of the path followed by the beam. In such cases, the standard multipole expansion (1) must be modified to give a field that satisfies
Maxwell's equations. For completeness, we would like to have a set of modes that can be used to describe threedimensional electric and magnetic fields in a coordinate system based on a curved reference trajectory and an efficient method for integrating the equations of motion for particles moving through these fields. In this paper, we present a suitable set of modes for static electric and magnetic fields and an explicit symplectic integrator for tracking particles through a given field (i.e., a field represented by a certain set of coefficients). The mode decomposition that we use is based on solutions to Laplace's equation in toroidal coordinates; the explicit symplectic integrator is developed following the method of $\mathrm{Wu}$, Forest, and Robin [3].

\section{DEFINITIONS}

We consider a particle of charge $q$ moving (at a relativistic velocity $v$ ) through a static electromagnetic field described by a scalar potential $\Phi$ and a vector potential $\mathbf{A}=\left(A_{x}, A_{y}, A_{s}\right)$. The Hamiltonian for the motion of the particle is [4]

$$
H=\frac{\delta}{\beta_{0}}-(1+h x) \sqrt{\left(\delta+\frac{1}{\beta_{0}}-\frac{q \Phi}{c P_{0}}\right)^{2}-\left(p_{x}-a_{x}\right)^{2}-\left(p_{y}-a_{y}\right)^{2}-\frac{1}{\beta_{0}^{2} \gamma_{0}^{2}}}-(1+h x) a_{s},
$$

where a particle with the chosen reference momentum $P_{0}$ has velocity $\beta_{0} c$ and relativistic factor $\gamma_{0}=\left(1-\beta_{0}^{2}\right)^{-1 / 2}$ and the scaled vector potential $\mathbf{a}=\left(a_{x}, a_{y}, a_{s}\right)=q \mathbf{A} / P_{0}$. The independent variable for the system is $s$, corresponding to distance along a reference trajectory. The reference trajectory follows the arc of a circle (in the plane perpendicular to $y$ ) with radius $\rho=1 / h$. At any point along the reference trajectory, the coordinates $x$ and $y$ describe (respectively) the horizontal and vertical position of the particle in a plane perpendicular to the reference trajectory. The longitudinal coordinate is defined as

$$
z=\frac{s}{\beta_{0}}-c t,
$$

where the particle arrives at position $s$ along the reference trajectory at time $t$ (and we can assume that, for the reference particle, $s=0$ at time $t=0$ ).

The momenta conjugate to the coordinates $x$ and $y$ are

$$
p_{x}=\frac{\gamma m v_{x}}{P_{0}}+a_{x}, \quad p_{y}=\frac{\gamma m v_{y}}{P_{0}}+a_{y},
$$

where $\gamma$ is the relativistic factor of the particle, $m$ is the mass, and $v_{x}$ and $v_{y}$ are the components of the velocity parallel to the $x$ and $y$ axes, respectively. The longitudinal conjugate momentum is

$$
\delta=\frac{E}{c P_{0}}-\frac{1}{\beta_{0}},
$$

where $E=\gamma m c^{2}+q \Phi$ is the total energy of the particle. To simplify some of the formulas, we introduce the "scaled" scalar potential $\phi$, defined by

$$
\phi=\frac{q \Phi}{c P_{0}}
$$

\section{DERIVATION OF THE SYMPLECTIC INTEGRATOR}

Our method follows the technique of $\mathrm{Wu}$, Forest, and Robin [3]. We first extend the phase space by introducing a new independent variable $\sigma$ so that $s$ is now a dynamical variable with conjugate momentum $p_{s}$. The Hamiltonian describing the motion of a particle through an electrostatic field with scaled potential $\phi=\phi(x, y, s)$ and magnetic field described by a scaled potential $\mathbf{a}=\left(a_{x}, a_{y}, a_{s}\right)$ is now 


$$
H^{\prime}=p_{s}+\frac{\delta}{\beta_{0}}-(1+h x) \sqrt{\left(\delta+\frac{1}{\beta_{0}}-\phi\right)^{2}-\left(p_{x}-a_{x}\right)^{2}-\left(p_{y}-a_{y}\right)^{2}-\frac{1}{\beta_{0}^{2} \gamma_{0}^{2}}}-(1+h x) a_{s} .
$$

We shall consider the special case where the magnetic field has a uniform vertical field component, which can be represented by a component of the vector potential:

$$
a_{s}=-k_{0} x+\frac{k_{0} h x^{2}}{2(1+h x)},
$$

where $k_{0}=q B_{0} / P_{0}$ for a magnetic field of strength $B_{0}$. If the field is correctly matched to the curvature of the reference trajectory (so that the reference trajectory is a possible physical trajectory of a particle with momentum $P_{0}$ ), then $h=k_{0}$. Other components of the magnetic field can be included in the components $a_{x}$ and $a_{y}$ of the vector potential.

We assume that the dynamical variables take small values so that we can approximate the Hamiltonian by expanding the square root to some order in the dynamical variables. In the conventional paraxial approximation, the expansion is made to second order. Here, we expand to third order and obtain

$$
H^{\prime} \approx H_{1 s}+H_{1 y}+H_{1 x}+H_{2}+H_{3}-1,
$$

where

$$
\begin{gathered}
H_{1 s}=p_{s}+\left(k_{0}-h\right) x+\frac{1}{2} h k_{0} x^{2}, \\
H_{1 y}=\frac{1}{2}\left(1+h x-\frac{\delta}{\beta_{0}}\right)\left(p_{y}-a_{y}\right)^{2}, \\
H_{1 x}=\frac{1}{2}\left(1+h x-\frac{\delta}{\beta_{0}}\right)\left(p_{x}-a_{x}\right)^{2}, \\
H_{2}=\frac{\phi}{\beta_{0}}+\frac{(\delta-\phi)^{2}}{2 \beta_{0}^{2} \gamma_{0}^{2}}\left(1+h x-\frac{\delta-\phi}{\beta_{0}}\right)-\frac{\delta-\phi}{\beta_{0}} h x, \\
H_{3}=\frac{\phi}{2 \beta_{0}}\left[\left(p_{x}-a_{x}\right)^{2}+\left(p_{y}-a_{y}\right)^{2}\right] .
\end{gathered}
$$

It should be noted that the values of the dynamical variables depend on the choice of gauge for the electromagnetic potentials: It should be borne in mind, therefore, that the choice of gauge may affect the accuracy of the expansion given in Eqs. (10)-(14). Also, the expansion and splitting of the Hamiltonian may be done in different ways. For example, in cases where the energy $E$ of a particle is constant, then $\delta$ may be treated as a parameter rather than a variable so a more accurate dependence on $\delta$ may be retained in the expansion.
Viewed as a Hamiltonian in its own right, the term $H_{1 s}$ is integrable, but this is not the case for the other terms, $H_{1 y}$, $H_{1 x}, H_{2}$, or $H_{3}$. However, by making appropriate canonical transformations to new variables, we can express $H_{1 y}, H_{1 x}$, and $H_{2}$ in integrable form. Assuming that the choice of gauge is such that the potential $\phi$ has no zeroth-order (constant) term, then $\mathrm{H}_{3}$ is of the order of 3 (or higher) in the dynamical variables; we assume we can drop this term (with some loss of accuracy in the solution to the equations of motion). We can then construct an explicit symplectic integrator as follows. The map for a step corresponding to a change $\Delta \sigma$ in the independent variable $\sigma$ is represented by

$$
e^{-\Delta \sigma: H:} \approx e^{-(\Delta \sigma / 2): H_{1}:} e^{-\Delta \sigma: H_{2}:} e^{-(\Delta \sigma / 2): H_{1}:},
$$

where

$$
H_{1}=H_{1 s}+H_{1 y}+H_{1 x} .
$$

Continuing the process,

$$
\begin{aligned}
& e^{-(\Delta \sigma / 2): H_{1}:} \\
& \quad \approx e^{-(\Delta \sigma / 4): H_{1 s}+H_{1 y}:} e^{-(\Delta \sigma / 2): H_{1 x}:} e^{-(\Delta \sigma / 4): H_{1 s}+H_{1 y}:},
\end{aligned}
$$

and finally

$$
e^{-(\Delta \sigma / 4): H_{1 s}+H_{1 y}:} \approx e^{-(\Delta \sigma / 8): H_{1 s}:} e^{-(\Delta \sigma / 4): H_{1 y}:} e^{-(\Delta \sigma / 8): H_{1 s}:} .
$$

The transformations associated with the generator $H_{1 s}$ are

$$
\begin{gathered}
e^{-(\Delta \sigma / 8): H_{1 s}:} s=s+\frac{\Delta \sigma}{8}, \\
e^{-(\Delta \sigma / 8): H_{1 s}:} p_{x}=p_{x}-\frac{\Delta \sigma}{8}\left(k_{0}-h+k_{0} h x\right),
\end{gathered}
$$

with the transformations of all other variables (not shown explicitly) corresponding to the identity.

Now consider $H_{1 y}$. To find an explicit form for the transformation generated by $H_{1 y}$, we first consider a transformation to new variables, defined by a mixedvariable generating function:

$$
F_{y}\left(X_{i}, p_{i} ; \sigma\right)=I_{Y}-X p_{x}-Y p_{y}-Z \delta-S p_{s},
$$

where $X_{i}=(X, Y, Z, S)$ are the new coordinates, $p_{i}=$ $\left(p_{x}, p_{y}, \delta, p_{s}\right)$ are the original momenta, and $I_{Y}$ is defined by 


$$
I_{Y}=\int_{0}^{Y} a_{y}(X, \bar{Y}, S) d \bar{Y}
$$

In Goldstein's nomenclature [5], $F_{y}\left(X_{i}, p_{i} ; \sigma\right)$ is a mixedvariable generating function of the third kind. The new coordinates $(X, Y, Z, S)$ are identical to the original coordinates $(x, y, z, s)$, since

$$
x=-\frac{\partial F_{y}}{\partial p_{x}}=X,
$$

and similarly for $y, z$, and $s$. The new momenta are

$$
\begin{gathered}
P_{X}=-\frac{\partial F_{y}}{\partial X}=p_{x}-\frac{\partial I_{Y}}{\partial X}, \\
P_{Y}=-\frac{\partial F_{y}}{\partial Y}=p_{y}-a_{y}, \\
P_{S}=-\frac{\partial F_{y}}{\partial S}=p_{s}-\frac{\partial I_{Y}}{\partial S},
\end{gathered}
$$

and

$$
P_{Z}=\delta
$$

In terms of the new variables, $H_{1 y}$ can be written

$$
H_{1 y}=\frac{1}{2}\left(1+h X-\frac{P_{Z}}{\beta_{0}}\right) P_{Y}^{2} .
$$

Viewed as a Hamiltonian, $H_{1 y}$ is integrable. The transformations (generated by $H_{1 y}$ ) of the dynamical variables are

$$
\begin{gathered}
e^{-(\Delta \sigma / 4): H_{1 y}: P_{X}}=P_{X}-\frac{\Delta \sigma}{8} h P_{Y}^{2}, \\
e^{-(\Delta \sigma / 4): H_{1 y}}: Y=Y+\frac{\Delta \sigma}{4}\left(1+h X-\frac{P_{Z}}{\beta_{0}}\right) P_{Y}, \\
e^{-(\Delta \sigma / 4): H_{1 y}:} Z=Z-\frac{\Delta \sigma}{8 \beta_{0}} P_{Y}^{2} .
\end{gathered}
$$

Again, the transformations of all other variables (i.e., for those variables not shown explicitly above) are given by the identity transformation. To apply the transformation $e^{-(\Delta \sigma / 4): H_{1 y}}$, we first transform from the original variables to a set of new variables using (24)-(26); we then apply the transformations (29)-(31) and finally transform back to the original variables using the inverse of the transformations (24)-(26). Note that, although the new momenta $P_{Y}$ and $P_{Z}$ do not change under the transformation generated by $H_{1 y}$, there will be changes in the original momenta $p_{y}$ and $p_{s}$ because of the change in the coordinate $Y$. The change in $Y$ means that the inverse of transformations (24)-(26) have to be calculated at a different point from the original transformations. Thus,

$$
\begin{aligned}
e^{-(\Delta \sigma / 4): H_{1 y}:} p_{x}= & p_{x}-\frac{\Delta \sigma}{8} h\left[p_{y}-a_{y}\left(x, y_{0}, s\right)\right]^{2} \\
& +\int_{y_{0}}^{y_{1}} \frac{\partial}{\partial x} a_{y}(x, \bar{y}, s) d \bar{y}, \\
e^{-(\Delta \sigma / 4): H_{1 y}:} p_{y}= & p_{y}+a_{y}\left(x, y_{1}, s\right)-a_{y}\left(x, y_{0}, s\right),
\end{aligned}
$$

where $y_{0}$ and $y_{1}$ correspond to the initial and final values, respectively, of the coordinate $y$ under the transformation $e^{-(\Delta \sigma / 4): H_{1 y}:}$. There is also a change in $p_{s}$, but this has no effect on the dynamics. In summary, to apply the transformation $e^{-(\Delta \sigma / 4): H_{1 y}}$, we need to evaluate $a_{y}$ (at the initial value of the coordinate $y=y_{0}$ and at the final value of the coordinate $y=y_{1}$ ) and the integral (with respect to $y$ ) of the derivative of $a_{y}$ (with respect to $x$ ).

In Sec. IV B, we give analytical expressions for the components of the vector potential, based on a threedimensional "multipole" decomposition of a magnetic field in a region with a curved reference trajectory. It is also possible to write down expressions for the derivatives of the vector potential; however, the integral in (32) needs to be performed numerically. Although this will make a significant contribution to the computational cost for each step in the tracking calculation, in most cases the integral should converge reasonably quickly given that the derivative of the potential (which is related to the field strength) should vary slowly over the range of the integral (corresponding to the change in the $y$ coordinate over the tracking step).

The transformation with generator $H_{1 x}$ may be handled in a similar way to that generated by $H_{1 y}$, by first transforming to new variables. For the case of $H_{1 x}$, we use the mixed-variable generating function

$$
F_{x}\left(X_{i}, p_{i} ; \sigma\right)=I_{X}-X p_{x}-Y p_{y}-Z \delta-S p_{s},
$$

where

$$
I_{X}=\int_{0}^{X} a_{x}(\bar{X}, Y, S) d \bar{X}
$$

Note that the new variables in this case (coordinates $X, Y$, $Z$, and $S$ and momenta $P_{X}, P_{Y}, P_{Z}$, and $P_{S}$ ) are formally different from the variables in the previous case; but, to avoid introducing further notation, we use the same symbols. The transformations (generated by $H_{1 x}$ ) of the dynamical variables are

$$
\begin{aligned}
& e^{-(\Delta \sigma / 2): H_{1 x}: x=} \frac{\Delta \sigma}{2}\left(1-\frac{\delta}{\beta_{0}}\right)\left(1+\frac{\Delta \sigma}{8} h P_{X}\right) P_{X} \\
&+\left(1+\frac{\Delta \sigma}{4} h P_{X}\right)^{2} x, \\
& e^{-(\Delta \sigma / 2): H_{1 x}}: p_{x}=\frac{P_{X}}{1+\frac{\Delta \sigma}{4} h P_{X}}+a_{x}\left(x_{1}, y, s\right),
\end{aligned}
$$




$$
\begin{gathered}
e^{-(\Delta \sigma / 2): H_{1 x}:} p_{y}=p_{y}+\int_{x_{0}}^{x_{1}} \frac{\partial}{\partial y} a_{x}(\bar{x}, y, s) d \bar{x}, \\
e^{-(\Delta \sigma / 2): H_{1 x}: z}=z-\frac{\Delta \sigma}{4 \beta_{0}} \frac{P_{X}^{2}}{\left(1+\frac{\Delta \sigma}{4} h P_{X}\right)},
\end{gathered}
$$

where

$$
P_{X}=p_{x}-a_{x}\left(x_{0}, y, s\right)
$$

and $x_{0}$ and $x_{1}$ are the values of $x$ before and after the transformation $e^{-(\Delta \sigma / 2): H_{1 x} \text { : }}$, respectively. The variables $y$ and $\delta$ are unchanged by the transformation.

Finally, we find explicit expressions for the transformation with generator $H_{2}$ by again first transforming to new variables. In this case, we use a mixed-variable generating function:

$$
F_{3}^{\prime}\left(X_{i}^{\prime}, p_{i} ; \sigma\right)=\phi\left(X^{\prime}, Y^{\prime}, S^{\prime}\right) Z^{\prime}-X^{\prime} p_{x}-Y^{\prime} p_{y}-Z^{\prime} \delta-S^{\prime} p_{s},
$$

where $X_{i}^{\prime}=\left(X^{\prime}, Y^{\prime}, Z^{\prime}, S^{\prime}\right)$ are the new coordinates and $p_{i}=\left(p_{x}, p_{y}, \delta, p_{s}\right)$ are the original momenta. The new coordinates are identical to the original coordinates, since

$$
x=-\frac{\partial F_{3}^{\prime}}{\partial p_{x}}=X^{\prime},
$$

and similarly for $y, z$, and $s$. The new momenta are

$$
\begin{aligned}
& P_{X}^{\prime}=-\frac{\partial F_{3}^{\prime}}{\partial X^{\prime}}=p_{x}-\frac{\partial \phi}{\partial X^{\prime}} Z^{\prime}, \\
& P_{Y}^{\prime}=-\frac{\partial F_{3}^{\prime}}{\partial Y^{\prime}}=p_{y}-\frac{\partial \phi}{\partial Y^{\prime}} Z^{\prime} \\
& P_{S}^{\prime}=-\frac{\partial F_{3}^{\prime}}{\partial S^{\prime}}=p_{s}-\frac{\partial \phi}{\partial S^{\prime}} Z^{\prime}
\end{aligned}
$$

and

$$
P_{Z}^{\prime}=\delta-\phi
$$

In terms of the new variables, $H_{2}$ can be written

$$
H_{2}=\frac{\phi}{\beta_{0}}+\frac{P_{Z}^{\prime 2}}{2 \beta_{0}^{2} \gamma_{0}^{2}}\left(1+h X^{\prime}-\frac{P_{Z}^{\prime}}{\beta_{0}}\right)-\frac{h}{\beta_{0}} X^{\prime} P_{Z}^{\prime},
$$

which is an integrable Hamiltonian, leading to the transformations

$$
e^{-\Delta \sigma: H_{2}:} P_{X}^{\prime}=P_{X}^{\prime}-\frac{\Delta \sigma}{\beta_{0}} \frac{\partial \phi}{\partial X^{\prime}}-\Delta \sigma \frac{h P_{Z}^{\prime 2}}{2 \beta_{0}^{2} \gamma_{0}^{2}}+\Delta \sigma \frac{h}{\beta_{0}} P_{Z}^{\prime},
$$

$$
\begin{gathered}
e^{-\Delta \sigma: H_{2}:} P_{Y}^{\prime}=P_{Y}^{\prime}-\frac{\Delta \sigma}{\beta_{0}} \frac{\partial \phi}{\partial Y^{\prime}}, \\
e^{-\Delta \sigma: H_{2}: P_{S}^{\prime}}=P_{S}^{\prime}-\frac{\Delta \sigma}{\beta_{0}} \frac{\partial \phi}{\partial S^{\prime}}, \\
e^{-\Delta \sigma: H_{2}: Z^{\prime}=} Z^{\prime}-\frac{\Delta \sigma}{\beta_{0}} h X^{\prime} \\
+\Delta \sigma \frac{P_{Z}^{\prime}}{\beta_{0}^{2} \gamma_{0}^{2}}\left(1+h X^{\prime}-\frac{3 P_{Z}^{\prime}}{2 \beta_{0}}\right) .
\end{gathered}
$$

Again, transformations of the variables not given explicitly above are equal to the identity.

\section{IV. $s$-DEPENDENT FIELDS IN TOROIDAL COORDINATES}

Applying the symplectic integrator described in Sec. III involves derivatives of the scalar potential and derivatives and integrals of the vector potential. It is therefore helpful to have analytic representations of the scalar and vector potentials, from which expressions for the derivatives and integrals may be found. In practice, however, only a purely numerical representation of the potentials may be available (giving, for example, the values of the potentials on a grid of discrete points over some region of space). With a straight reference trajectory $(h=0)$, it is possible to fit the coefficients of series representations of the potentials, for example, using generalized gradients [1]; the series representation gives the functional dependence of the potential on the coordinates, and this therefore provides a suitable representation for applying the integrator.

A similar approach is possible in the case that the reference trajectory has some nonzero curvature. Expressions for "curvilinear multipoles" (multipole fields around curved reference trajectories) have been given by McMillan and others [6-10] and have been implemented in the tracking code Bmad [11]. However, the available expressions are not ideal for use where the potential is given in a purely numerical form. In much of the previous work, the multipoles are expressed in terms of the transverse Cartesian coordinates $x$ and $y$ : Obtaining the multipole coefficients then involves fitting polynomials to the numerical data along either the $x$ or the $y$ axis [12]. The nature of the potential (which satisfies Laplace's equation) is such that residuals to the fit will grow exponentially with distance from the line along which the fit is performed. A more robust approach is based on fitting to a surface bounding some region of space enclosing the reference trajectory: Within the surface, the residuals decrease exponentially with distance from the surface. Although the residuals will still grow exponentially outside the region enclosed by the surface, if the surface is chosen appropriately, then the enclosed region will cover the volume of interest for particle tracking. 


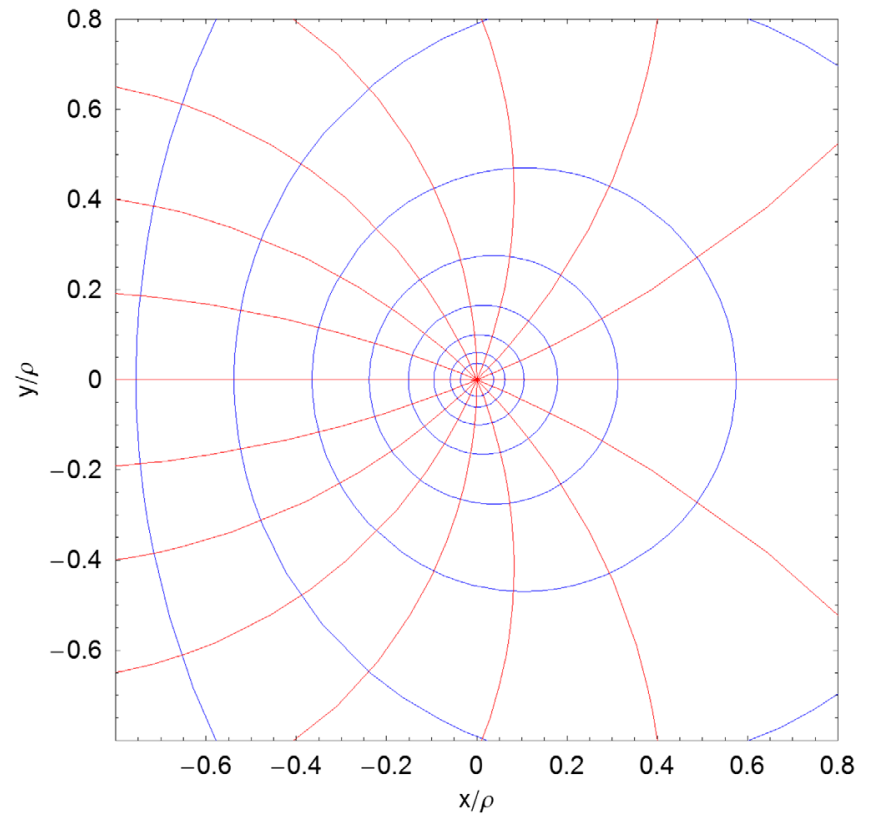

FIG. 1. Toroidal coordinates. The red curves show lines of constant $v$ from 0 to $2 \pi$. The blue curves show lines of constant value for the coordinate $u$ in the range $0.5-4$ in steps of 0.5 , with $0 \leq v<2 \pi$. Larger values of $u$ give circles of smaller diameter; in the limit $u \rightarrow \infty$, the circles converge towards the reference trajectory $x=y=0$.

To obtain a multipole decomposition based on fitting numerical data on a surface, it is convenient in the case of a curved reference trajectory to work in toroidal coordinates $[9,13,14]$. The coordinates in the transverse plane are illustrated in Fig. 1. The toroidal coordinates $u$ and $v$ are related to the accelerator coordinates $x$ and $y$ (Cartesian coordinates in a plane perpendicular to the reference trajectory) by

$$
\begin{gathered}
x=\rho\left(\frac{\sinh (u)}{\cosh (u)-\cos (v)}-1\right), \\
y=\frac{\rho \sin (v)}{\cosh (u)-\cos (v)},
\end{gathered}
$$

where $\rho=1 / h$ is the radius of curvature of the reference trajectory. The longitudinal coordinate $s$ (the distance along the reference trajectory) is related to the toroidal coordinate $\theta$ by

$$
s=\rho \theta .
$$

A surface enclosing the reference trajectory can be defined by specifying a fixed value $u_{\text {ref }}$ for the coordinate $u$ : A surface defined by $u=u_{\text {ref }}$ for $0 \leq v<2 \pi$ and $0 \leq \theta<2 \pi$ resembles a torus. If numerical field data are available for the scalar and vector potentials on such a surface, then it is possible to fit the coefficients of series expansions for the scalar and vector potentials (up to some desired order) to the data. This produces expressions that are suitable for use in the explicit symplectic integrator described in Sec. III. We first discuss the case of the scalar potential and then extend the results to the vector potential.

A potential drawback of the use of toroidal coordinates is the fact that a point on the reference trajectory (which has, by definition, $x=y=0$ ) is described in toroidal coordinates by the limit $u \rightarrow \infty$. This raises the possibility of numerical instabilities when calculating the potentials or tracking particles close to the reference trajectory, and some care may be needed in the practical implementation of the relevant formulas. However, in realistic cases the expressions for the potentials are well behaved in the limit $u \rightarrow \infty$, as should be expected for physical quantities. The examples given in Sec. V are based on an implementation of the formulas for calculating the potentials and tracking particles in Mathematica 5.0 [15], and we have not encountered any problems from numerical instabilities when using this code.

\section{A. Scalar potential in toroidal coordinates}

In terms of the toroidal coordinates, an harmonic potential (such that $\nabla^{2} \phi=0$ ) may be written $[13,16]$

$$
\phi=\sum_{m, n=-\infty}^{\infty} f_{m n} \phi_{m n}
$$

where the $f_{m n}$ are coefficients representing the strength of a multipole component $\phi_{m n}$. The multipole components are given by

$$
\phi_{m n}=(-i)^{m} \mathcal{C}(u, v) P_{n-(1 / 2)}^{-|m|}(\operatorname{coth}(u)) e^{i m v} e^{i n \theta},
$$

where $P_{\nu}^{\mu}(\xi)$ is an associated Legendre polynomial of the first kind and

$$
\mathcal{C}(u, v)=\sqrt{\frac{\cosh (u)-\cos (v)}{\sinh (u)}}=\sqrt{\frac{\rho}{x+\rho}} .
$$

An algorithm for the computation of the associated Legendre polynomials with positive $\mu$ has been presented by Segura and Gil [17]; values for negative $\mu$ are readily obtained using [18]

$$
P_{\nu}^{-\mu}(\xi)=\frac{\Gamma(\nu-\mu+1)}{\Gamma(\nu+\mu+1)}\left(P_{\nu}^{\mu}(\xi)-\frac{2}{\pi} e^{-i \mu \pi} \sin (\mu \pi) Q_{\nu}^{\mu}(\xi)\right),
$$

where $Q_{\nu}^{\mu}(\xi)$ is an associated Legendre polynomial of the second kind. Note that, for integer $\mu$ (which is the case of interest here), the term in $Q_{\nu}^{\mu}(\xi)$ in (58) vanishes. 
We shall show in Sec. IV C that each component $\phi_{m n}$ has properties that may be expected of a multipole of the order of $m$, with $m=1$ corresponding to a dipole, $m=2$ a quadrupole, and so on. Note that a normal dipole deflects a particle horizontally, whereas a skew dipole deflects a particle vertically.

Given numerical data for a potential $\phi(u, v, \theta)$, the coefficients $f_{m n}$ may be obtained from

$f_{m n}=\frac{1}{N_{m n}} \int_{0}^{2 \pi} d v \int_{0}^{2 \pi} d \theta \frac{e^{-i m v} e^{-i n \theta} \phi\left(u_{\mathrm{ref}}, v, \theta\right)}{\sqrt{\cosh \left(u_{\mathrm{ref}}\right)-\cos (v)}}$,

where $u_{\text {ref }}$ is a fixed value of $u$ that defines the surface (enclosing the reference trajectory, $x=y=0$ ) on which the fit to the numerical data is performed and $N_{m n}$ is a normalizing factor:

$$
N_{m n}=(-i)^{m} 4 \pi^{2} \frac{P_{n-(1 / 2)}^{-|m|}\left(\operatorname{coth}\left(u_{\mathrm{ref}}\right)\right)}{\sqrt{\sinh \left(u_{\mathrm{ref}}\right)}} .
$$

As an alternative to calculating the coefficients $f_{m n}$ from the scalar potential, they may be calculated from the electric field components. The electric field is derived from the potential by

$$
\mathbf{E}=\left(E_{u}, E_{v}, E_{\theta}\right)=-\nabla \Phi=-\frac{c P_{0}}{q} \nabla \phi
$$

The $E_{v}$ component of the field (tangential to a line defined by fixed values of $u$ and $\theta$ ) is given by

$$
E_{v}=-\frac{c P_{0}}{q} \sum_{m, n} f_{m n} \frac{(-i)^{m}}{\rho}\left\{\frac{1}{2} \sin (v)+i m[\cosh (u)-\cos (v)]\right\} \mathcal{C}(u, v) P_{n-(1 / 2)}^{-|m|}(\operatorname{coth}(u)) e^{i m v} e^{i n \theta}
$$

The coefficients $f_{m n}$ can then be found from the values of $E_{v}$ on a surface $u=u_{\text {ref }}$ :

$$
f_{m n}=\frac{1}{N_{m n}^{\prime}} \int_{0}^{2 \pi} d v \int_{0}^{2 \pi} d \theta \frac{e^{-i m v} e^{-i n \theta} E_{v}\left(u_{\mathrm{ref}}, v, \theta\right)}{\left\{\frac{1}{2} \sin (v)+i m\left[\cosh \left(u_{\mathrm{ref}}\right)-\cos (v)\right]\right\} \mathcal{C}\left(u_{\mathrm{ref}}, v\right)},
$$

where

$$
N_{m n}^{\prime}=-\frac{(-i)^{m}}{\rho} 4 \pi^{2} P_{n-(1 / 2)}^{-|m|}\left(\operatorname{coth}\left(u_{\mathrm{ref}}\right)\right)
$$

To apply the symplectic integrator described in Sec. III, we need the derivatives of the potential with respect to the Cartesian coordinates. The derivates can be obtained from

$$
\begin{aligned}
& \frac{\partial \phi}{\partial x}=\frac{\partial \phi}{\partial u} \frac{\partial u}{\partial x}+\frac{\partial \phi}{\partial v} \frac{\partial v}{\partial x}, \\
& \frac{\partial \phi}{\partial y}=\frac{\partial \phi}{\partial u} \frac{\partial u}{\partial y}+\frac{\partial \phi}{\partial v} \frac{\partial v}{\partial y},
\end{aligned}
$$

and

$$
\frac{\partial \phi}{\partial s}=\frac{\partial \phi}{\partial \theta} \frac{\partial \theta}{\partial s}=\frac{1}{\rho} \frac{\partial \phi}{\partial \theta}
$$

For a given multipole component (56), the derivatives with respect to the toroidal coordinates $u$ and $v$ are

$$
\begin{aligned}
\frac{\partial \phi_{m n}}{\partial u}= & (-i)^{m}\left[\left(n \operatorname{coth}(u)+\frac{1}{2 \mathcal{C}(u, v)}\right) P_{n-(1 / 2)}^{-|m|}(\operatorname{coth}(u))\right. \\
& \left.-\left(|m|+n+\frac{1}{2}\right) \mathcal{C}(u, v) P_{n+(1 / 2)}^{-|m|}(\operatorname{coth}(u))\right] \\
& \times e^{i m v} e^{i n \theta}
\end{aligned}
$$

and

$$
\begin{aligned}
\frac{\partial \phi_{m n}}{\partial v}= & (-i)^{m}\left(\frac{\sin (v)}{2 \sinh (u) \mathcal{C}(u, v)}+i m \mathcal{C}(u, v)\right) \\
& \times P_{n-(1 / 2)}^{-|m|}(\operatorname{coth}(u)) e^{i m v} e^{i n \theta} .
\end{aligned}
$$

Finally, we need the derivatives of the toroidal coordinates $(u, v)$ with respect to the Cartesian coordinates $(x, y)$. The toroidal coordinates can be expressed in terms of the Cartesian coordinates as follows:

$$
u-i v=2 \operatorname{coth}^{-1}\left(1+\frac{x+i y}{\rho}\right) .
$$

We then find 


$$
\begin{aligned}
\frac{\partial u}{\partial x} & =\frac{\partial v}{\partial y}=\frac{-2 \rho\left[(2 \rho+x) x-y^{2}\right]}{\left(x^{2}+y^{2}\right)\left[(2 \rho+x)^{2}+y^{2}\right]} \\
& =\frac{1}{\rho}[1-\cosh (u) \cos (v)]
\end{aligned}
$$

and

$$
\begin{aligned}
\frac{\partial u}{\partial y} & =-\frac{\partial v}{\partial x}=\frac{-4 \rho(\rho+x) y}{\left(x^{2}+y^{2}\right)\left[(2 \rho+x)^{2}+y^{2}\right]} \\
& =\frac{1}{\rho} \sinh (u) \sin (v) .
\end{aligned}
$$

The derivatives of the potential with respect to the Cartesian coordinates can be found by using Eqs. (68), (69), (71), and (72) in Eqs. (65) and (66). Tracking a particle through a field described by a scalar potential can then be achieved by using the potential and its derivatives (with respect to $x$ and $y$ ) in the symplectic integrator described in Sec. III.

\section{B. Vector potential in toroidal coordinates}

To apply the explicit symplectic integrator to a particle moving through a magnetic field, we need expressions for the components of the vector potential. Since we address the case of a curved reference trajectory, we assume that the magnetic field has a (normal) dipole component derived from the longitudinal component $a_{s}$ of the vector potential (8). Other components of the magnetic field (corresponding to quadrupole or higher-order multipole components) may be derived from the transverse components of the vector potential. In toroidal coordinates, these components may be expressed as follows:

$$
\begin{aligned}
& a_{u}=i \sinh (u) \sum_{m, n=-\infty}^{\infty} \frac{\alpha_{m n}}{n} \frac{\partial \phi_{m n}}{\partial v} \\
& a_{v}=-i \sinh (u) \sum_{m, n=-\infty}^{\infty} \frac{\alpha_{m n}}{n} \frac{\partial \phi_{m n}}{\partial u}
\end{aligned}
$$

where the functions $\phi_{m n}$ are given by (56). In the case that $a_{\theta}=0$ [i.e., the longitudinal component of the vector potential is zero so that $k_{0}=0$ in (8)] and $\alpha_{m n}=f_{m n}$ for all $m, n$, it is found that

$$
\nabla \times \mathbf{a}=-\nabla \phi,
$$

with $\phi$ given by (55). Hence, the magnetic field derived from the vector potential $\mathbf{a}=\left(a_{u}, a_{v}, 0\right)$ with components (in toroidal coordinates) given by (73) and (74) has the same form as the electric field derived from the scalar potential $\phi$ given by (55).

To apply the symplectic integrator described in Sec. III, we require the components of the vector potential in Cartesian coordinates and their derivatives. Given the components $\left(a_{u}, a_{v}\right)$ in toroidal coordinates, the components $\left(a_{x}, a_{y}\right)$ in Cartesian coordinates are obtained from

$$
\begin{aligned}
& a_{x}=\frac{1}{N} \frac{\partial x}{\partial u} a_{u}+\frac{1}{N} \frac{\partial x}{\partial v} a_{v}=\frac{[1-\cosh (u) \cos (v)] a_{u}-\sinh (u) \sin (v) a_{v}}{\cosh (u)-\cos (v)}, \\
& a_{y}=\frac{1}{N} \frac{\partial y}{\partial u} a_{u}+\frac{1}{N} \frac{\partial y}{\partial v} a_{v}=-\frac{[1-\cosh (u) \cos (v)] a_{v}+\sinh (u) \sin (v) a_{u}}{\cosh (u)-\cos (v)},
\end{aligned}
$$

where the normalizing factor $N$ is

$$
\begin{aligned}
N & =\sqrt{\left(\frac{\partial x}{\partial u}\right)^{2}+\left(\frac{\partial y}{\partial u}\right)^{2}}=\sqrt{\left(\frac{\partial x}{\partial v}\right)^{2}+\left(\frac{\partial y}{\partial v}\right)^{2}} \\
& =\frac{\rho}{\cosh (u)-\cos (v)} .
\end{aligned}
$$

The derivatives of $a_{x}$ and $a_{y}$ with respect to the Cartesian coordinates $x$ and $y$ can be expressed in terms of the derivatives with respect to the toroidal coordinates $u$ and $v$ :

$$
\begin{aligned}
& \frac{\partial a_{x}}{\partial y}=\frac{\partial u}{\partial y} \frac{\partial a_{x}}{\partial u}+\frac{\partial v}{\partial y} \frac{\partial a_{x}}{\partial v} \\
& \frac{\partial a_{y}}{\partial x}=\frac{\partial u}{\partial x} \frac{\partial a_{y}}{\partial u}+\frac{\partial v}{\partial x} \frac{\partial a_{y}}{\partial v}
\end{aligned}
$$

Given (73) and (74), the derivatives of $a_{x}$ and $a_{y}$ with respect to the toroidal coordinates may be found from the second derivatives of the scalar potential:

$$
\begin{aligned}
\frac{\partial^{2} \phi_{m n}}{\partial u^{2}}= & (-i)^{m}\left[\frac{c_{1}}{16 \sinh ^{4}(u) \mathcal{C}(u, v)^{3}} P_{n-(1 / 2)}^{-|m|}(\operatorname{coth}(u))+\frac{c_{2}}{\sinh ^{2}(u) \mathcal{C}(u, v)}\left(|m|+n+\frac{1}{2}\right) P_{n+(1 / 2)}^{-|m|}(\operatorname{coth}(u))\right. \\
& \left.+\mathcal{C}(u, v)\left(|m|+n+\frac{1}{2}\right)\left(|m|+n+\frac{3}{2}\right) P_{n+(3 / 2)}^{-|m|}(\operatorname{coth}(u))\right] e^{i m v} e^{i n \theta},
\end{aligned}
$$




$$
\begin{gathered}
\frac{\partial^{2} \phi_{m n}}{\partial u \partial v}=(-i)^{m-1}\left[\frac{c_{3}}{4 \sinh ^{3}(u) \mathcal{C}(u, v)^{3}} P_{n-(1 / 2)}^{-|m|}(\operatorname{coth}(u))+\frac{c_{4}}{2 \sinh (u) \mathcal{C}(u, v)}\left(|m|+n+\frac{1}{2}\right) P_{n+(1 / 2)}^{-|m|}(\operatorname{coth}(u))\right] e^{i m v} e^{i n \theta} \\
\frac{\partial^{2} \phi_{m n}}{\partial v^{2}}=(-i)^{m} \frac{c_{5}}{\sinh (u) \mathcal{C}(u, v)} P_{n-(1 / 2)}^{-|m|}(\operatorname{coth}(u)) e^{i m v} e^{i n \theta}
\end{gathered}
$$

where

$$
\begin{gathered}
c_{1}=4\left[1-2 n(2 n-5)-\left(1+2 n+4 n^{2}\right) \cosh (2 u)\right] \cos (v) \cosh (u)+4 n\{6(n-1)+[n \cosh (2 u)+n-2] \cos (2 v)\} \\
+[5+4 n(7 n-3)] \sinh (u)^{2}+(1+2 n)^{2} \sinh (u) \sinh (3 u), \\
c_{2}=1+(1+2 n) \cos (v) \cosh (u)-2(1+n) \cosh ^{2}(u), \\
\left.c_{3}=2 m \cos (v)-i \sin (v)+i n \cosh (u) \sin (2 v)+m[4 n-1+2 n \cos (2 v)+(1+2 n) \cosh (2 u)]\right) \cosh (u) \\
+[i(1-2 n) \sin (v)-2 m(1+4 n) \cos (v)] \cosh ^{2}(u), \\
c_{4}=i \sin (v)-2 m[\cosh (u)-\cos (v)] \\
c_{5}=\frac{1}{2} \cos (v)-m^{2}[\cosh (u)-\cos (v)]+\left(i m-\frac{\sin (v)}{4[\cosh (u)-\cos (v)]}\right) \sin (v) .
\end{gathered}
$$

\section{Examples of multipole potentials in toroidal coordinates}

To illustrate the scalar potential given by (55), we consider the case that the potential is independent of the longitudinal coordinate $\theta$ : As a consequence, we need to include only a single longitudinal mode $n=0$ in the summation in (55). With a straight reference trajectory $(h=0)$, we expect a multipole potential to take the form

$$
\phi_{m}=\operatorname{Re}\left[C_{m}(x+i y)^{m}\right],
$$

where the real and imaginary parts of the coefficient $C_{m}$ determine the strengths of the normal and skew components of the field. Hence, in a normal multipole field of the order of $m$, the potential varies along the $x$ axis as

$$
\phi_{m}=\operatorname{Re}\left(C_{m}\right) x^{m}
$$

and along the $y$ axis as

$$
\phi_{m}= \begin{cases}\operatorname{Im}\left(C_{m}\right) y^{m} & \text { odd } m \\ \operatorname{Re}\left(C_{m}\right) y^{m} & \text { even } m\end{cases}
$$

With a curved reference trajectory, we expect to see similar behavior in the dependence of the potential for a given order of multipole on the $x$ and $y$ coordinates but with some difference from the dependence given in (89) arising from the curvature. One way to show a similarity between multipoles with straight and curved reference trajectories would be to expand the potential in the case of a multipole with a curved reference trajectory as a series in $x$ and $y$; unfortunately, the fact that the limit $x \rightarrow 0, y \rightarrow 0$ corresponds to $u \rightarrow \infty$ makes it problematic to obtain the appropriate series. However, we can plot the potential for a given order of (normal or skew) multipole as a function of $x$ and $y$ : Plots for dipoles, quadrupoles, and sextupoles are shown in Figs. 2 (normal multipoles) and 3 (skew multipoles).

From Fig. 2(top), for example, we see that for a normal dipole the potential has an approximately linear dependence on $x$. With a straight reference trajectory, we would expect the potential to be independent of $y$; however, the curvature of the reference trajectory introduces a secondorder dependence of the potential on $y$. In the case of a normal quadrupole (Fig. 2, middle), the potential has a (roughly) quadratic dependence on both $x$ and $y$ : This again corresponds to the behavior that we would expect in the case of a straight reference trajectory. Because the curvature of the reference trajectory breaks the symmetry between positive and negative values of $x$, the effect of the curvature is more evident in the dependence of the potential on $x$ than in the dependence of the potential on $y$. For a skew quadrupole (Fig. 3, middle), the potential with a straight 

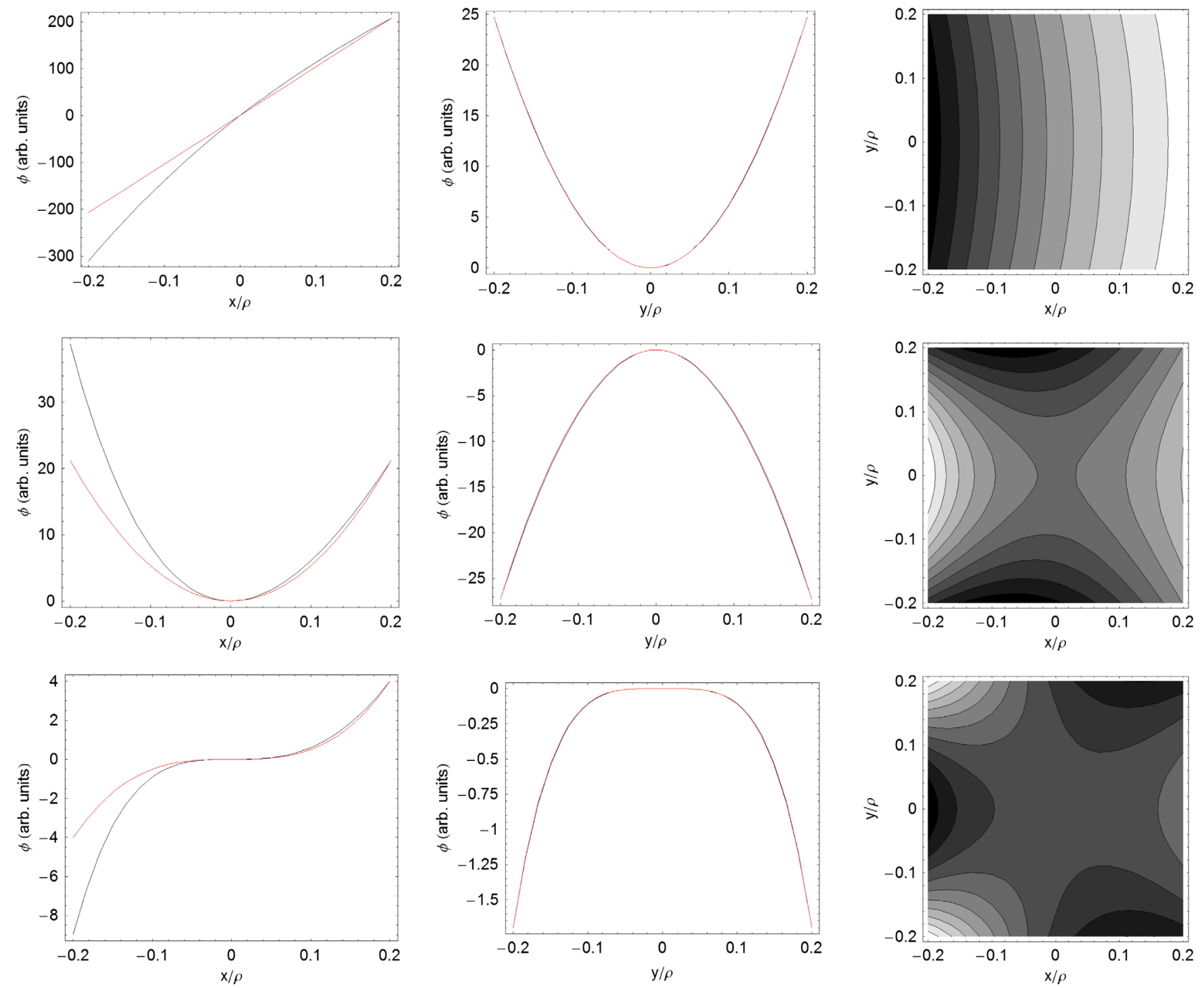

FIG. 2. Scalar potential in normal multipoles with a curved reference trajectory. Each row shows (top to bottom) the potential in a multipole of the order of $n=1$ (dipole), the order of $n=2$ (quadrupole), and the order of $n=3$ (sextupole). The left-hand and middle plots in each row show, respectively, the potential (black line) as a function of horizontal position $x$, with $y=0$, and as a function of vertical position $y$, with $x=0$. The red lines in the left-hand plots show curves $\phi \propto x^{n}$. The red lines in the middle plots show curves $\phi \propto y^{n+1}$ for odd $n$ and $\phi \propto y^{n}$ for even $n$. The right-hand plot in each row shows contours of constant potential in the plane perpendicular to the reference trajectory.

reference trajectory is exactly zero along the $x$ and $y$ axes. With a curved reference trajectory, the potential is zero along the $x$ axis (as required by symmetry), but there is a relatively weak fourth-order dependence of the potential on $y$ (with $x=0$ ). Other cases demonstrate the general behavior we would expect for a multipole potential in a straight coordinate system but with some differences arising from the curvature of the reference trajectory.

\section{TEST CASES}

To illustrate application of the explicit symplectic integrator presented in Sec. III, we consider three test cases: a curvilinear magnetic skew sextupole, a curvilinear electrostatic quadrupole, and the fringe-field region of an electrostatic quadrupole in the $g-2$ storage ring [19-22]. The first two cases are "artificial" in the sense that they are based on fields described by a small number of components; the third case is more realistic and uses field component coefficients fitted to numerical data obtained from a modeling code. In each case, we track a particle with some chosen initial conditions through the field using the explicit symplectic integrator. For comparison, we also integrate numerically the (Hamiltonian) equations of motion derived from the exact Hamiltonian (2). All calculations are performed in Mathematica 5.0 [15]; for 

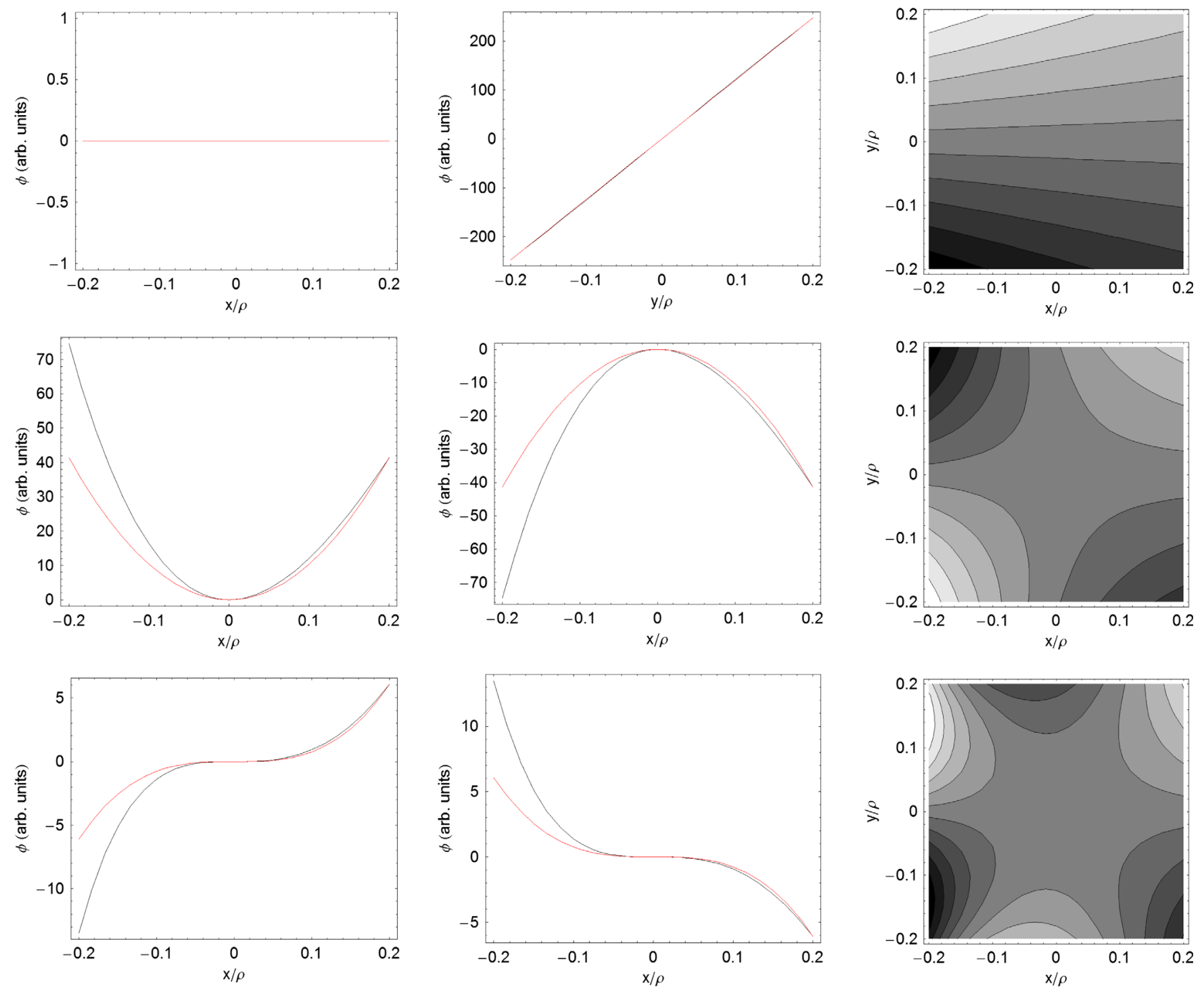

FIG. 3. Scalar potential in skew multipoles with a curved reference trajectory. Each row shows (top to bottom) the potential in a multipole of the order of $n=1$ (dipole), the order of $n=2$ (quadrupole), and the order of $n=3$ (sextupole). The left-hand and middle plots in the top row (dipole) show, respectively, the potential as a function of $x$, with $y=0$, and as a function of $y$, with $x=0$ (black line). In the middle and bottom rows (quadrupole and sextupole), the left-hand and middle plots show, respectively, the potential as a function of $x$, with $y=x \tan (\pi / 2 n)$, and as a function of $x$, with $y=-x \tan (\pi / 2 n)$ (black lines). The red lines in the left-hand and middle plots show curves $\phi \propto x^{n}$ (or $\phi \propto y^{n}$ in the top row, middle plot). The right-hand plot in each row shows contours of constant potential in the plane perpendicular to the reference trajectory.

numerical integration of the equations of motion derived from the Hamiltonian (2), we use the NDSolve function with default settings; although this provides a nonsymplectic integration, it should achieve good accuracy.

\section{A. Curvilinear magnetic skew sextupole}

As a first illustration of the explicit symplectic integrator presented in Sec. III, we consider the motion of a particle in an electric field with a (scaled) magnetic scalar potential given by

$$
\begin{gathered}
\phi=\phi_{0} \sqrt{\frac{\cosh (u)-\cos (v)}{\sinh (u)}}\left[\frac{1}{12} P_{12-(1 / 2)}^{-3}(\operatorname{coth}(u)) \sin (12 \theta)\right. \\
\left.-P_{1-(1 / 2)}^{-3}(\operatorname{coth}(u)) \sin (\theta)\right] \cos (3 v)
\end{gathered}
$$

The field derived from this potential has the characteristics of a skew sextupole field, as shown in Fig. 4. We choose the field strength such that $\phi_{0}=5 \times 10^{4}$ and use a radius of curvature for the reference trajectory $\rho=5 \mathrm{~m}$. A dipole 

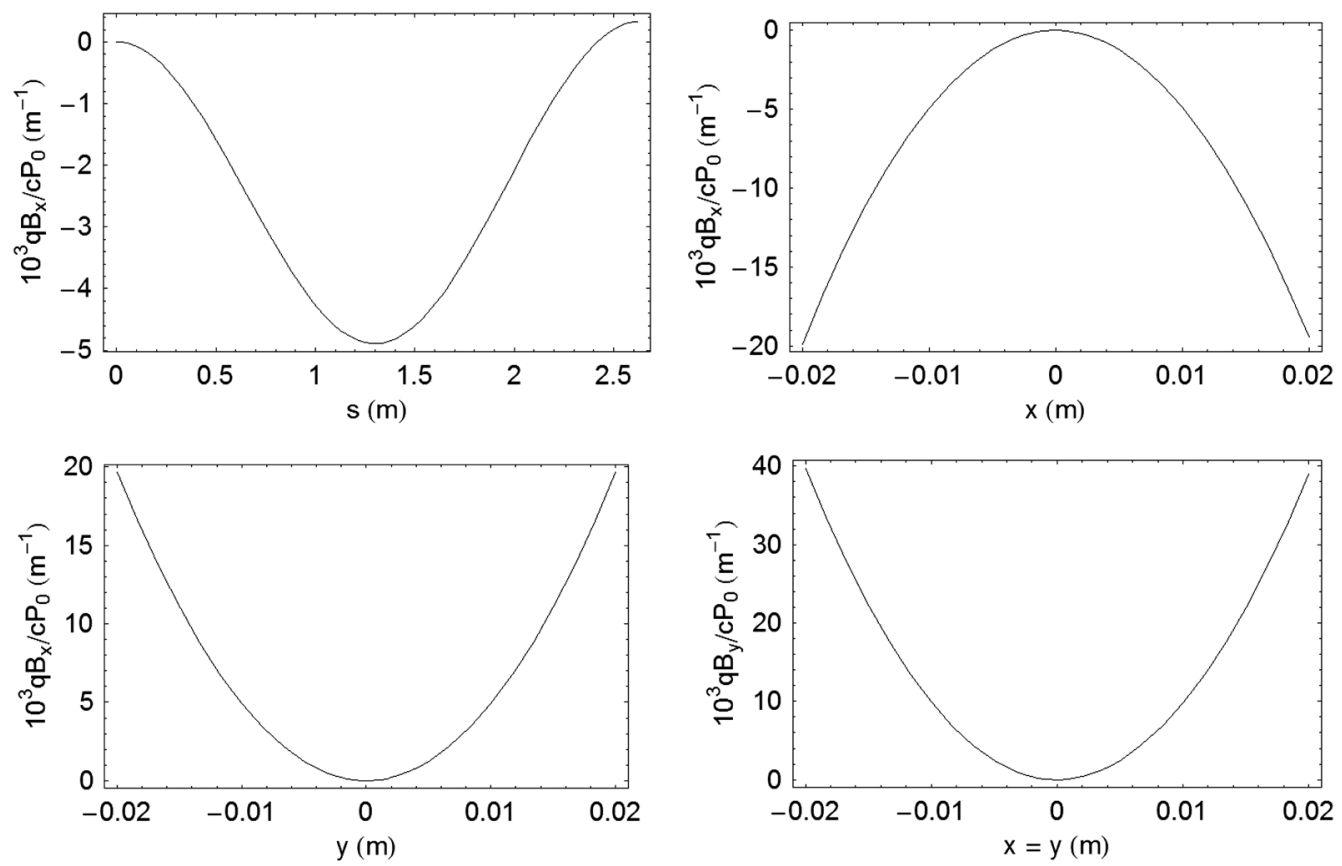

FIG. 4. Magnetic field in a curvilinear skew sextupole, derived from the scalar potential (92). Top left: $B_{x}$ as a function of $s$ for $x=10 \mathrm{~mm}$ and $y=0$. Top right: $B_{x}$ as a function of $x$ for $y=0$ and $s=\frac{\pi}{12} \rho$. Bottom left: $B_{x}$ as a function of $y$ for $x=0, s=\frac{\pi}{12} \rho$. Bottom right: $B_{y}$ along the line $x=y$, for $s=\frac{\pi}{12} \rho$. In each plot, the field is scaled by the beam rigidity.

magnetic field is included, represented by the longitudinal component of the vector potential (8), but with $k_{0}=1.05 / \rho$ so that there is a slight mismatch between the field and the curvature of the reference trajectory.

For the reference particle, we choose $\beta_{0}=0.8$, and the initial conditions for the particle to be tracked are

$$
\begin{aligned}
& \left(x, p_{x}, y, p_{y}, z, \delta\right) \\
& \quad=\left(1 \mathrm{~mm}, 4 \times 10^{-3}, 1 \mathrm{~mm},-0.1 \times 10^{-3}, 0,0.02\right) .
\end{aligned}
$$

We track the particle using the explicit symplectic integrator presented in Sec. III, from $s=0$ to $s=s_{\max }=\frac{\pi}{6} \rho$, with a step size of $\Delta \sigma=s_{\max } / 10$. The integration required in (32) is approximated by Simpson's rule:

$$
\begin{aligned}
\left.\int_{y_{0}}^{y_{1}} \frac{\partial a_{y}}{\partial x}\right|_{y=\bar{y}} d \bar{y} \approx \frac{y_{1}-y_{0}}{6} & \\
& \times\left(\left.\frac{\partial a_{y}}{\partial x}\right|_{y=y_{0}}+\left.4 \frac{\partial a_{y}}{\partial x}\right|_{y=(1 / 2)\left(y_{0}+y_{1}\right)}+\left.\frac{\partial a_{y}}{\partial x}\right|_{y=y_{1}}\right)
\end{aligned}
$$

where the derivative is evaluated in each case at the appropriate (fixed) values of $x$ and $s$ and at the indicated value of $y$. A similar approximation is made for the integration in (38). Although these approximations will lead to some symplectic error, this should be small for a small step size. In cases where symplecticity is important, more accurate integration routines can be used, though at a greater computational cost.
The tracking results are shown in Fig. 5. There is good agreement between the two integration methods.

\section{B. Curvilinear electrostatic quadrupole}

As a second illustration of the explicit symplectic integrator presented in Sec. III, we consider the motion of a particle in an electric field with (scaled) scalar potential given by

$$
\begin{aligned}
& \phi=\phi_{0} \sqrt{\frac{\cosh (u)-\cos (v)}{\sinh (u)}}\left[P_{12-(1 / 2)}^{-2}(\operatorname{coth}(u)) \cos (12 \theta)\right. \\
& \left.-P_{-(1 / 2)}^{-2}(\operatorname{coth}(u))\right] \cos (2 v) .
\end{aligned}
$$

This represents the potential for a "curvilinear" electrostatic quadrupole, with a strength that varies with the longitudinal position along the reference trajectory. The transverse and longitudinal variation of the field are described by $m=2$ and $n=12$ (respectively) in Eq. (55). The potential is illustrated in Fig. 6. We choose the field strength $\phi_{0}=200$ and use a radius of curvature for the reference trajectory $\rho=5 \mathrm{~m}$. We include a magnetic field, represented by the vector potential (8), but we introduce a small mismatch between the field and the curvature of the reference trajectory by setting $k_{0}=1.05 / \rho$.

For the reference particle, we choose $\beta_{0}=0.8$, and the initial conditions for the particle to be tracked are 

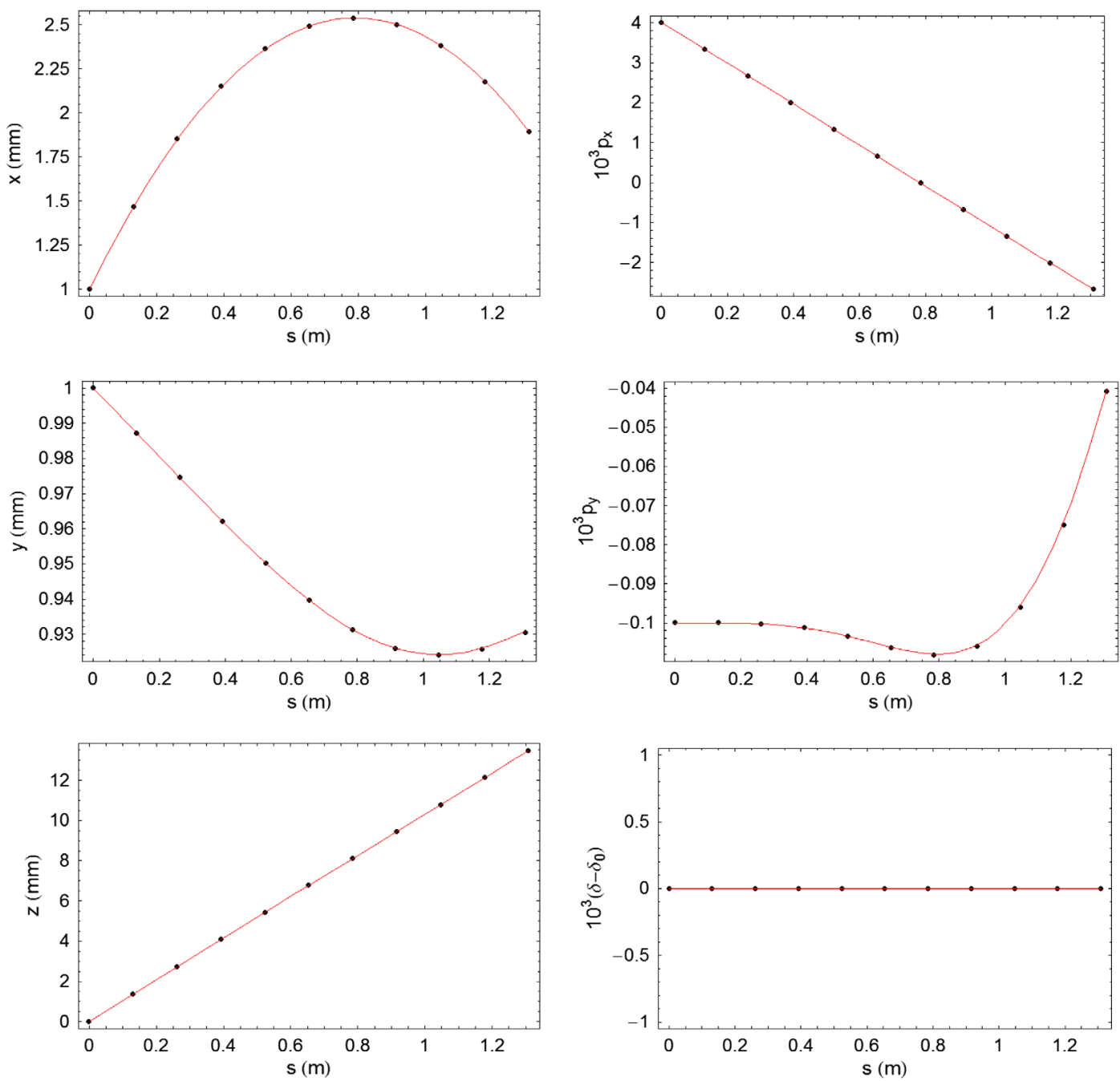

FIG. 5. Results of tracking a particle through a magnetic curvilinear skew sextupole, described by the magnetic scalar potential given in Eq. (92). The black points show the results from the explicit symplectic integrator presented in Sec. III. The red lines show the results of numerical integration of the equations of motion derived from the Hamiltonian (2).
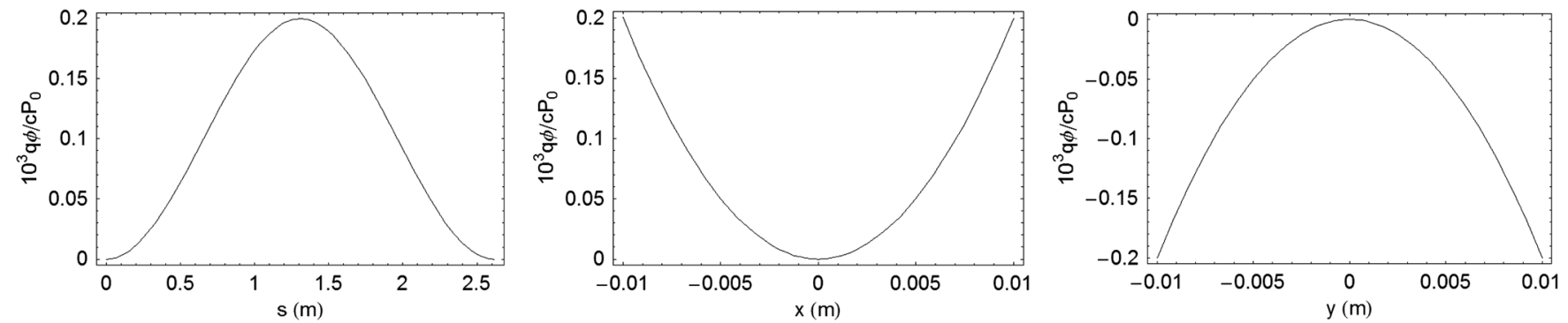

FIG. 6. Variation of the electrostatic potential (95) in a curvilinear quadrupole, as a function of the coordinates $s$ (left-hand plot, for $x=10 \mathrm{~mm}$ and $y=0$ ), $x$ (middle plot, for $y=0$ and $s=\frac{\pi}{12} \rho$ ), and $y$ (right-hand plot, for $x=0$ and $s=\frac{\pi}{12} \rho$ ).

$\left(x, p_{x}, y, p_{y}, z, \delta\right)=\left(2 \mathrm{~mm}, 0,1 \mathrm{~mm},-1.1 \times 10^{-3}, 0,0.02\right)$.

We track the particle using the explicit symplectic integrator presented in Sec. III, from $s=0$ to $s=s_{\max }=\frac{\pi}{6} \rho$, with a step size of $\Delta \sigma=s_{\max } / 40$. For comparison, we also integrate numerically the (Hamiltonian) equations of motion derived from the exact Hamiltonian (2). The tracking results are shown in Fig. 7, and again we see good agreement between the two integration methods. 

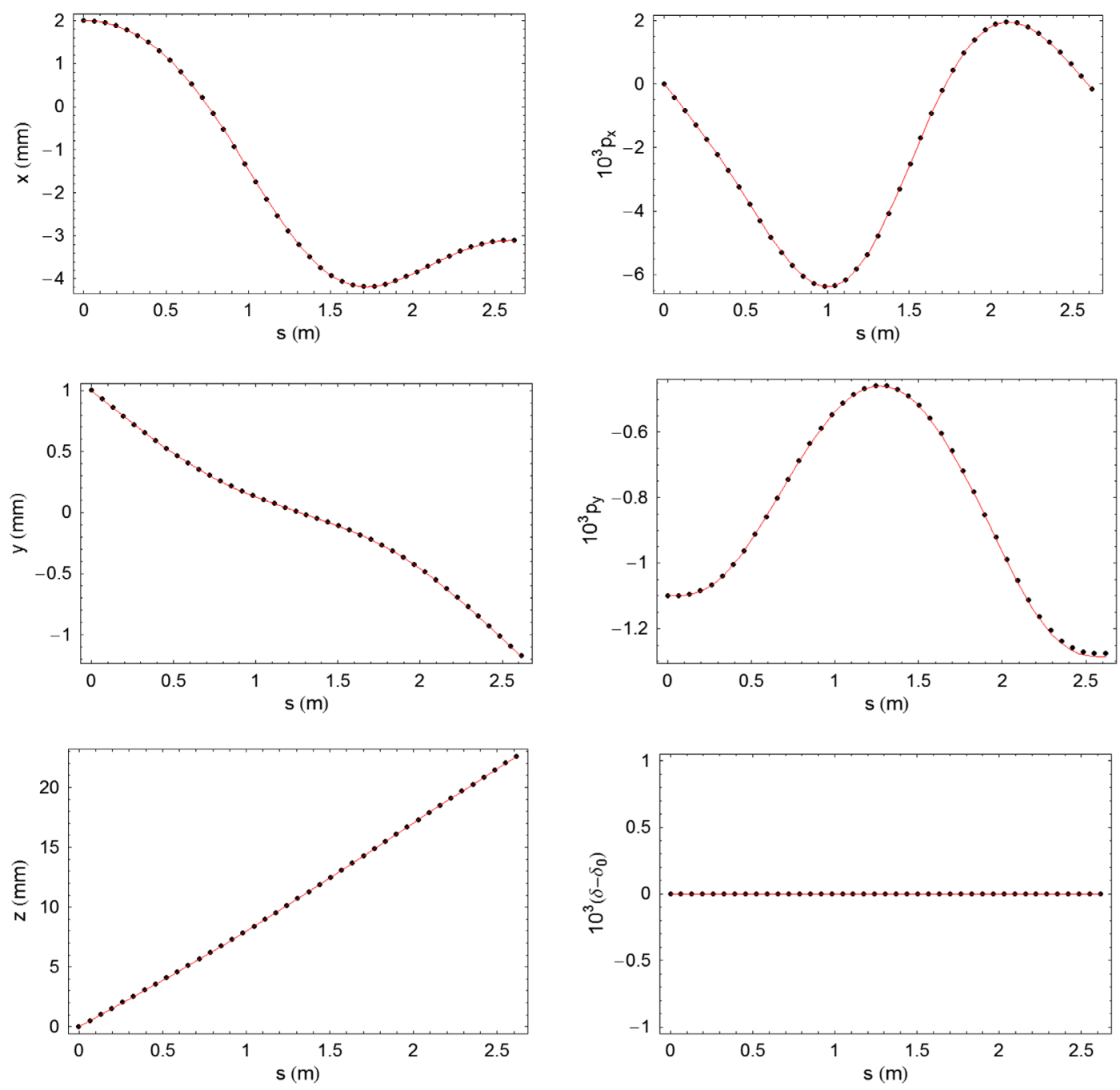

FIG. 7. Results of tracking a particle through the field of a curvilinear electrostatic quadrupole. The potential is given by Eq. (95). The black points show the results from the explicit symplectic integrator described in Sec. III. The red lines show the results from numerical integration of the equations of motion derived from the Hamiltonian (2).

\section{C. $g$ - 2 storage ring electrostatic quadrupole}

As a final example of application of the symplectic integrator, we consider the fringe-field regions of the electrostatic quadrupoles in the $g-2$ storage ring [19-22]. Values for the potential were calculated (using an finite element analysis code) at points on a uniform Cartesian grid; the values of the potential on a surface defined (in toroidal coordinates) by $u=u_{\text {ref }}=5.76$ were then obtained by (spline) interpolation. On the surface $u=u_{\text {ref }}$, we used 120 grid points in $v$, with $0 \leq v<2 \pi$, and 80 grid points in $\theta$, with $0<\theta \leq 2^{\circ}$ (such that the ends of the quadrupole electrodes are at approximately $\theta=1^{\circ}$ ). The reference radius for the coordinate system is taken to be the radius of curvature of the reference trajectory in the $g-2$ storage ring, $\rho=7.112 \mathrm{~m}$ : This is the radius of the closed orbit for muons with momentum $3.094 \mathrm{GeV} / c$. The value of $u=5.76$ then corresponds, for $v=0$, to a point with $x=0.045 \mathrm{~m}$ and $y=0$, in the conventional accelerator coordinate system, with the origin for the $x$ and $y$ coordinates on the reference trajectory.

Based on Eq. (55), coefficients $f_{m n}$ were calculated so that the potential on any grid point can be found from

$$
\begin{aligned}
\phi= & \sqrt{\frac{\cosh (u)-\cos (v)}{\sinh (u)}} \\
& \times \sum_{m, n} f_{m n} i^{m} P_{n^{\prime}-(1 / 2)}^{-m}(\operatorname{coth}(u)) \cos (m v) \sin \left(n^{\prime} \theta\right),
\end{aligned}
$$

where $n^{\prime}=n_{0}(2 n+1)$, with $n_{0}=45$ (so that $n=0$ corresponds to a sine function with the quarter period equal to $2^{\circ}$, i.e., the range of $\theta$ over which values for the potential are given). The values of $f_{m n}$ are obtained essentially by a discrete Fourier transform of the potential 


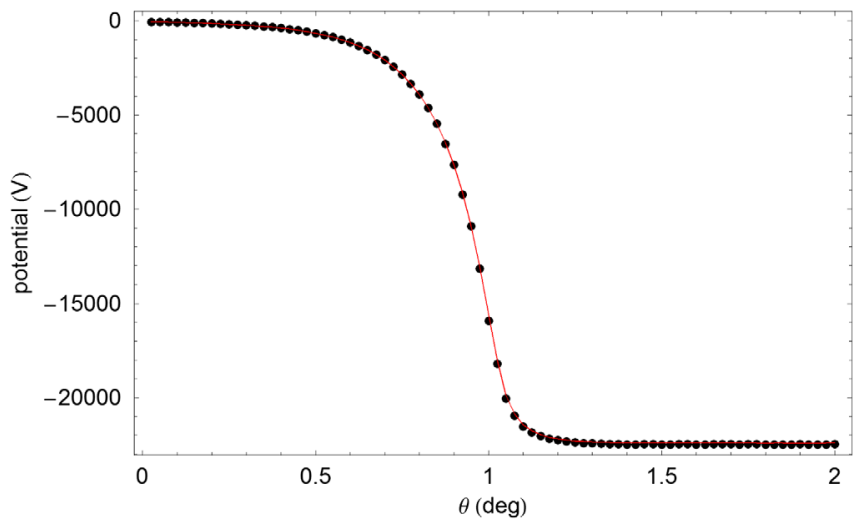

FIG. 8. Scalar potential in an electrostatic quadrupole in the $g-2$ storage ring. The potential is plotted as a function of toroidal coordinate $\theta$ at $u=u_{\text {ref }}$ and $v=0$. The black points show the original data points; the red line shows a fit using Eq. (97).

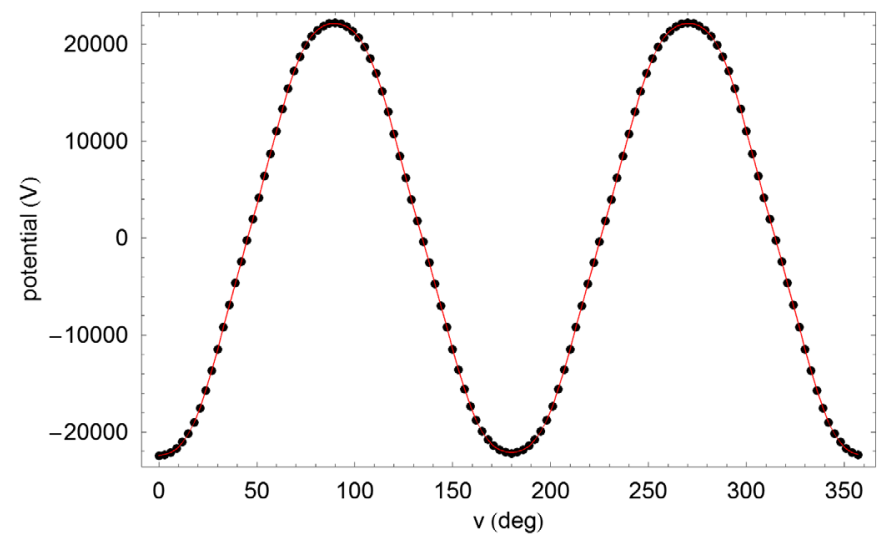

on the given grid points. Mode numbers $0 \leq m \leq 10$ and $0 \leq n \leq 79$ are used. The truncation in the azimuthal mode number $m$ (compared to the number of data points available) means that the data are not fitted perfectly; however, the contribution of modes (multipoles) of the order of $m>10$ is found to be small. Note that the dominant multipole is the quadrupole component, $m=2$.

The potential as a function of $\theta$ (at $u=u_{\text {ref }}$ and $v=0$ ) is shown in Fig. 8 and as a function of $v$ (at $\theta=2^{\circ}$ and at $\theta=0.25^{\circ}$, with $u=u_{\text {ref }}$ in both cases) in Fig. 9. In the right-hand plot in Fig. 9, we see that the variation of the potential with the "azimuthal" coordinate $v$ in the fringefield region (about $30 \mathrm{~mm}$ from the ends of the electrodes) is significantly distorted from a simple sine wave, indicating the presence of higher-order multipoles.

Using the coefficients $f_{m n}$, we can calculate the potential at any point within the surface on which the fit is performed. As an example, Fig. 10 shows the potential

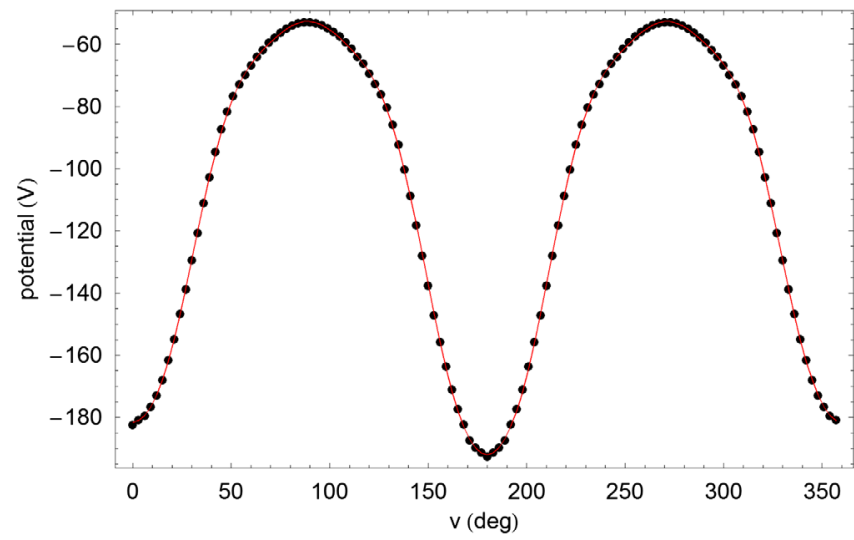

FIG. 9. Scalar potential in an electrostatic quadrupole in the $g-2$ storage ring. The potential is plotted as a function of toroidal coordinate $v$ at $\theta=2^{\circ}$ (left) and at $\theta=0.25^{\circ}$ (right), with $u=u_{\text {ref }}$ in both cases. The black points show the original data points; the red lines show fits using Eq. (97).
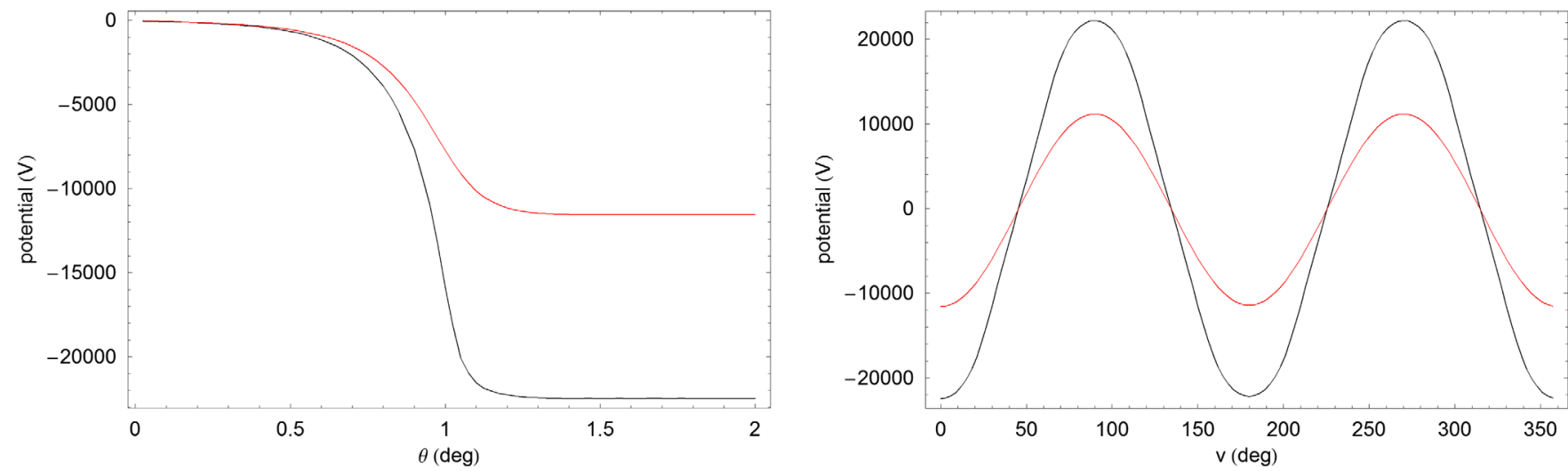

FIG. 10. Scalar potential in an electrostatic quadrupole in the $g-2$ storage ring. The potential is plotted as a function of toroidal coordinate $\theta$ at $v=0$ (left) and as a function of $v$ at $\theta=2^{\circ}$ (right). In each plot, the black line shows the potential at $u=u_{\text {ref }}=5.76$, and the red line shows the potential at $u=6.11$. At the larger value of $u$, the value of the coordinate $x$ is reduced by a factor of $\sqrt{2}$ compared to the value of $x$ at $u=u_{\text {ref }}$; the potential is a factor of 2 smaller at the larger value of $u$, as expected for a quadrupole field. 

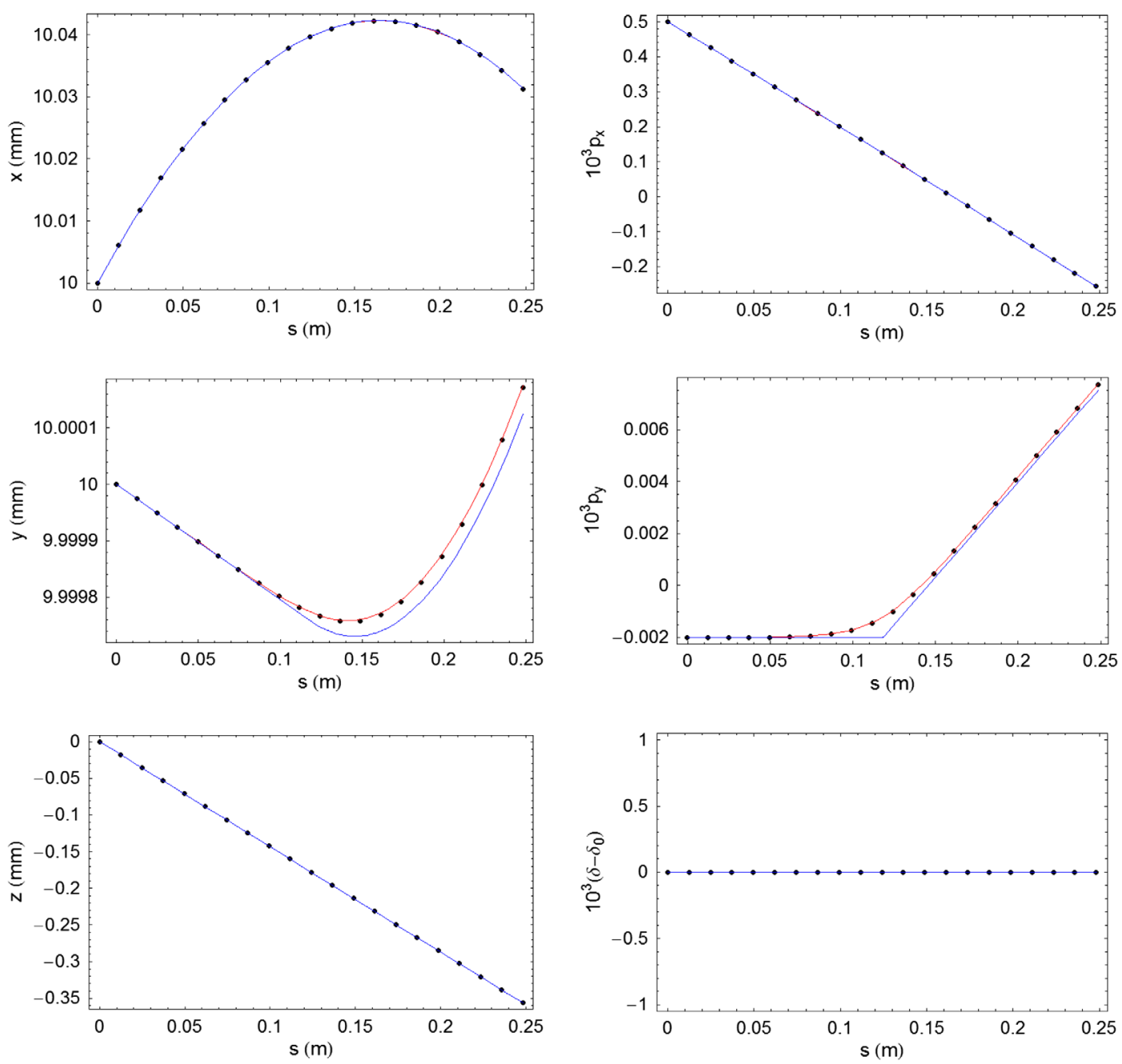

FIG. 11. Trajectory of a muon through the fringe-field region of an electrostatic quadrupole in the $g-2$ storage ring. The electrostatic potential is shown in Figs. 8 and 9. The reference momentum is $3.094 \mathrm{GeV} / c$, and the reference trajectory is the arc of a circle with a radius of $7.112 \mathrm{~m}$, determined by the magnetic field strength $B \approx 1.45 \mathrm{~T}$. The initial coordinates $\left(x, p_{x}, y, p_{y}, z, \delta\right)$ of the muon are $\left(10 \mathrm{~mm}, 5 \times 10^{-4}, 10 \mathrm{~mm},-2 \times 10^{-6}, 0,-0.02\right)$. The black points show the results from the symplectic integrator, with a step size of $12.4 \mathrm{~mm}$, i.e., a total of 20 steps. The red line shows the results of an integration using a (nonsymplectic) adaptive Runge-Kutta integration of the equations of motion in the same field. The blue line shows the results of a Runge-Kutta integration of the equations of motion through a region with the same magnetic field but with a hard-edge model for the electric field.

as a function of $\theta$ (for $v=0$ ) and as a function of $v$ (for $\theta=2^{\circ}$ ). In each plot, the black line shows the potential at $u=u_{\mathrm{ref}}=5.76$, and the red line shows the potential at $u=6.11$ : The larger value of $u$ corresponds to a value of $x$ that is a factor of $\sqrt{2}$ smaller than the value of $x$ at $u=u_{\text {ref }}$ so that the potential (for a pure quadrupole) is expected to be smaller by a factor of 2 . The expected behavior of the potential (as a function of $u$ ) is indeed what we observe.

Tracking a particle through the fringe field of an electrostatic quadrupole using the symplectic integrator described in Sec. III requires the derivatives of the potential with respect to the accelerator coordinates, $x, y$, and $s$. The derivatives can be calculated (at any point within the surface used to fit the coefficients $f_{m n}$ for the given potential) using Eq. (97), together with (71) and (72).
Some example results from tracking a muon through the fringe field are shown in Fig. 11. The black points in Fig. 11 show the muon trajectory calculated using the symplectic integrator for the detailed fringe-field model, i.e., the model based on the numerical data for the scalar potential. The red line shows the results of an integration using a (nonsymplectic) adaptive Runge-Kutta integration of the equations of motion in the same field. The blue line shows the results of a Runge-Kutta integration of the equations of motion through a region with the same magnetic field but with a "hard-edge" model for the electric field. The hard-edge model is constructed so that the scalar potential is zero up to a point $s=s_{1}$ and is given simply by $\phi=\frac{1}{2} k_{1}\left(x^{2}-y^{2}\right)$ for $s>s_{1}$. The value of $k_{1}$ is chosen to correspond to the focusing potential in the body of the 
quadrupole found from the numerical data for the scalar potential. The point $s_{1}$ is chosen so that the integrated gradient $\int_{0}^{s_{\max }} k_{1} d s$ in the hard-edge model is equal to the integrated gradient in the fringe-field model.

There is good agreement between the symplectic integrator and the Runge-Kutta integrator for the detailed fringe-field model. The horizontal motion is dominated by the magnetic field, which is the same for both the detailed fringe-field model and the hard-edge model of the quadrupole, so the results from the two models show no significant difference in this case. There is some small but observable difference between the detailed fringe-field model and the hard-edge model for the vertical motion. The change in the vertical momentum after integrating through the full region is approximately the same in both cases: This is expected, since the length of the quadrupole field in the hard-edge model was chosen to give the same integrated focusing strength as the detailed fringe-field model. However, the fact that the change in the vertical momentum occurs at a discrete point in the hard-edge model leads to a slightly larger difference between the models in the vertical coordinate at the end of the integration. It is unclear what impact this may have on the beam dynamics in the storage ring, but it is possible that it may lead to an observable effect over a sufficiently large number of turns.

\section{ACKNOWLEDGMENTS}

We thank Bruno Muratori for useful discussions and members of the $g-2$ Collaboration for help and support with studies of the $g-2$ electrostatic quadrupoles. In particular, we are grateful to Wanwei Wu for providing field data. This work was supported by the Science and Technology Facilities Council, United Kingdom, through a grant to the Cockcroft Institute.

[1] M. Venturini and A. J. Dragt, Accurate computation of transfer maps from magnetic field data, Nucl. Instrum. Methods Phys. Res., Sect. A 427, 387 (1999).

[2] B. Muratori, J. K. Jones, and A. Wolski, Analytical expressions for fringe fields in multipole magnets, Phys. Rev. ST Accel. Beams 18, 064001 (2015).

[3] Y. K. Wu, E. Forest, and D. S. Robin, Explicit symplectic integrator for $s$-dependent static magnetic field, Phys. Rev. E 68, 046502 (2003).

[4] A. Wolski, Beam Dynamics in High Energy Particle Accelerators (Imperial College, London, 2014).

[5] H. Goldstein, C. P. Poole, Jr., and J. L. Safko, Classical Mechanics, 3rd ed. (Addison-Wesley, Reading, MA, 2001).
[6] E. M. McMillan, Multipoles in cylindrical coordinates, Nucl. Instrum. Methods 127, 471 (1975).

[7] T. Zolkin, Sector magnets or transverse electromagnetic fields in cylindrical coordinates, Phys. Rev. Accel. Beams 20, 043501 (2017).

[8] P. Schnizer, E. Fischer, and B. Schnizer, Cylindrical circular and elliptical, toroidal circular and elliptical multipoles fields, potentials and their measurement for accelerator magnets, arXiv:1410.8090.

[9] P. Schnizer, Advanced Multipoles for Accelerator Magnets (Springer, New York, 2017).

[10] L. Brouwer, S. Caspi, D. Robin, and W. Wan, 3D toroidal field multipoles for curved accelerator magnets, in Proceedings of PAC2013, Pasadena, CA, USA (JACoW, Pasadena, CA, USA, 2013), pp. 907-909.

[11] D. Sagan, The Bmad Reference Manual, Sec. 14.3, https:// www.classe.cornell.edu/bmad/manual.html, pp. 252-254.

[12] A. T. Herrod, S. Jones, A. Wolski, I. R. Bailey, and M. Korostelev, Modelling of curvilinear electrostatic multipoles in the Fermilab muon g-2 storage ring, in Proceedings of IPAC2017, Copenhagen, 2017 (JACoW, Copenhagen, Denmark, 2017), pp. 3837-3839.

[13] G. Arfken, Mathematical Methods for Physicists, 2nd ed. (Academic, New York, 1970), Sec. 2.13, pp. 112-115.

[14] E. W. Weissten, Toroidal coordinates, MathWorld-A Wolfram Web Resource, http://mathworld.wolfram.com/ ToroidalCoordinates.html.

[15] Wolfram Mathematica, https://www.wolfram.com/ mathematica/.

[16] E. W. Weissten, Laplace's equation-Toroidal coordinates, MathWorld-A Wolfram Web Resource, http://mathworld .wolfram.com/LaplacesEquationToroidalCoordinates.html.

[17] J. Segura and A. Gil, Evaluation of toroidal harmonics, Comput. Phys. Commun. 124, 104 (2000).

[18] Handbook of Mathematical Functions with Formulas, Graphs and Mathematical Tables, edited by M. Abramowitz and I. Stegun (Martino, Eastford, CT, 2014).

[19] Muon $g$-2 Collaboration, Muon $g-2$ Technical Design Report No. Fermilab-FN-0992-E, arXiv:1501.06858.

[20] Muon $g-2$ Collaboration, Muon $g-2$ Technical Design Report No. Fermilab-FN-0992-E, Chap. 13: The electrostatic quadrupoles (ESQ) and beam collimators, arXiv: 1501.06858, pp. 395-422.

[21] W. Wu and B. Quinn, Beam dynamics in g-2 storage ring, in Proceedings of IPAC2017, Copenhagen, Denmark, 2017 (JACoW, Copenhagen, Denmark, 2017), pp. 817-819.

[22] Y. K. Semertzidis, G. Bennett, E. Efstathiadis, F. Krienen, R. Larsen, Y. Y. Lee, W. M. Morse, Y. Orlov, C. S. Ozben, B. L. Roberts, L. P. Snydstrup, and D. S. Warburton, The Brookhaven muon $(g-2)$ storage ring high voltage quadrupoles, Nucl. Instrum. Methods Phys. Res., Sect. A 503, 458 (2003). 